\title{
CONICAL SINGULAR POINTS OF DIFFEOMORPHISMS
}

\author{
BY WILLIAM HUEBSCH AND MARSTON MORSE
}

Communicated by Edwin Moise, May 27, 1961

1. Introduction. The Schoenflies extension $\Lambda_{\phi}$ of a differentiable mapping $\phi$, constructed in the proof of Theorem 2.1 of [1], has at most a differential singularity of conical type (to be defined). This fact has far-reaching consequences which are reflected in the theorems of [2]. Theorem 1.1 below is one of these consequences. No proof of Theorem 1.1 is given here.

Let $S$ be an $(n-1)$-sphere in a euclidean $n$-space $E$ and let $J S$ be the closed $n$-ball in $E$ bounded by $S$.

TheOREM 1.1. Let $z$ be an arbitrary point of $S$. A real analytic diffeomorphism $f$ of $S$ into $E$ admits a homeomorphic extension, $F$, defined over a set $Z \cup z$, where $Z$ is some open neighborhood of $J S-z$, and $F \mid Z$ is a real analytic diffeomorphism of $Z$ into $E$.

This extension $F$ of $f$ defines an analytic diffeomorphism of its domain of definition with $z$ deleted, and a homeomorphism with $z$ included. $F$ has no singularity on the interior of $S$, or on $S$, except at most at $z$.

We continue with a detailed exposition leading to a proof of Theorem 2.1.

Notation. Let $E$ be the euclidean $n$-space of points (or vectors) $x$ with rectangular coordinates $\left(x_{1}, \cdots, x_{n}\right)$. Let $\|x\|$ be the distance of $x$ from the origin $O$. Set

$$
S=\{x \mid\|x\|=1\} .
$$

If $M$ is a topological $(n-1)$-sphere in $E, \stackrel{J}{J} M$ shall denote the open interior of $M$. The complement of a subset $Y$ of $E$ will be denoted by $C Y$. We use diff as an abbreviation of diffeomorphism.

$A C_{z}^{m}$-diff, $m>0$. Let $x \rightarrow G(x)$ be a homeomorphism into $E$ of an open neighborhood $X$ of a point $z \in E$; if $G \mid(X-z)$ is a $C^{m}$-diff into $E$, $G$ will be called a $C_{z}^{m}$-diff of $X$ into $E$.

An admissible cone $K_{z}$. Let $K_{z}$ be a closed $n$-cone in $E$ with vertex $z$, and with sections orthogonal to $A$ which are closed $(n-1)$-balls whose centers are on $A$. The cone $K_{z}$ is determined by $z, A$ and any one of its orthogonal sections meeting $A-z$.

$A$ conical point $z$ of $G$. Let $G$ be a $C_{z}^{m}$-diff into $E$ of an open neighborhood $X$ of $z$. The point $z$ will be said to be a conical point of $G$ and 
$K_{z}$ a cone of singular approach to $z$ if there exists a $C^{m}$-diff $\zeta$ into $E$ of some open neighborhood $U \subset X$ of $z$ such that

$$
G(x)=\zeta(x) \quad\left(x \in U-K_{z}\right) .
$$

On the supposition that $z$ is a conical point of $G$ we prove the following lemma.

Lemma 1.1. (i) If $\mu$ is a $C^{m}$-diff into $X$ of an open neighborhood $Y$ of a point $y$ such that $\mu(y)=z$, then $G \mu$ is a $C_{y}^{m}$-diff of $Y$ into $E$ with conical point $y$.

(ii) If $\theta$ is a $C^{m}$-diff of $G(X)$ into $E$, then $\theta G$ is a $C_{z}^{m}$-diff of $X$ into $E$ with conical point $z$.

Proof OF (i). Suppose that $G$ is represented on $U-K_{z}$ as in (1.2). Let $W \subset Y$ be an open neighborhood of $y$ so small that $\mu(W) \subset U$, and for some admissible cone $K_{y}$

$$
\mu\left(W-K_{y}\right) \subset U-K_{z} .
$$

Put $\mu \mid W=\mu_{1}$. Then $\zeta \mu_{1}$ is a $C^{m}$-diff of $W$ into $E$, and it follows from (1.2) and (1.3) that

$$
(G \mu)(x)=\left(\zeta \mu_{1}\right)(x) \quad\left(x \in W-K_{y}\right) .
$$

This partial representation (1.4) of $G \mu$ shows that $y$ is a conical point of the $C_{y}^{m}$-diff $G \mu$. This establishes (i).

The proof of (ii) is immediate.

2. The principal theorem. In [1] there is given a $C^{m}$-diff $\phi$ into $E$ of a "shell" neighborhood $\delta_{a}$ of $S$ such that $\phi$ carries points of $\delta_{a}$ interior to $S$ into points of $E$ interior to the manifold $\phi(S)$, and it is shown (see [1, Theorem 2.1]) that there exists an open neighborhood $U \subset \delta_{a}$ of $S$, a point $z \in \dot{J} S$ and a $C_{z}^{m}$-diff $\Lambda_{\phi}$ of $U \cup \dot{J} S$ into $E$ which extends $\phi \mid U$. The construction of $\Lambda_{\phi}$ is carried through in [1] for the case in which $\phi$ is special, in the sense that $\phi$ reduces to the identity in the neighborhood of a point $Q$ of $S$. In this paper we supplement Theorem 2.1 of [1] by proving the following.

THEOREM 2.1. The $C_{z}^{m}$-diff $\Lambda_{\phi}$, as constructed in [1] for a "special" $C^{m}$-diff $\phi$, has z as conical point.

To prove this theorem we review the necessary parts of the construction of $\Lambda_{\phi}$ in [1].

The relevant subsets of $E$. Let $K$ be the open $n$-cube [1, p. 273]

$$
K=\left\{x \mid-1<x_{i}<1 ; i=1, \cdots, n\right\} .
$$


Let $K^{\prime}$ be the subrectangle of $K$ on which $x_{n}<0$. Subrectangles $H^{\prime} \supset L^{\prime} \supset G^{\prime}$ of $K^{\prime}$ are introduced with faces parallel to those of $K^{\prime}$, of which $H^{\prime}$ and $L^{\prime}$ are open and $G^{\prime}$ closed, while

$$
\mathrm{Cl} H^{\prime} \subset K^{\prime}, \quad \mathrm{Cl} L^{\prime} \subset H^{\prime} .
$$

Let $D=\left\{x \mid-1<x_{i}<9 ; i=1, \cdots, n\right\}$ and set $P=(8,0, \cdots, 0)$. $A$ radial mapping $R$ of $E$ onto $E$. $R$ is defined by the equations

$$
y_{1}-8=\frac{x_{1}-8}{2} ; \quad y_{j}=\frac{x_{j}}{2} \quad(j=2, \cdots, n)
$$

and leaves $P$ fixed. If $R^{r}$ is the $r$-fold iterate of $R$ and $R^{0}$ the identity, the space $E$ admits a trivial partition

$$
E=\left[\bigcup_{r=0}^{\infty} R^{r}(K)\right] \cup P \cup A \quad \text { (Cf. (5.1) of [1]) }
$$

provided $A$ is suitably chosen.

The mapping $T$. If $B$ is a bounded subset of $E$, Int $B$ shall denote the smallest $n$-rectangle $\Pi$ in $E$ with faces parallel to the coordinate $(n-1)$-planes and such that $\Pi \supset B$. In $\S 6$ of [1], a $C^{\infty}$-diff $T$ of $E$ onto $E$ is defined. For us the essential properties of $T$ are that

$$
R T(\bar{K}) \cap T(\bar{K})=\varnothing, \quad T(\bar{K}) \subset \operatorname{Int}(\bar{K} \cup R \bar{K}) .
$$

One sets $T_{r+1}=R^{r} T, r=0,1, \cdots$.

The cone $K_{P}$. Let $K_{P}$ be the smallest admissible cone with vertex $P$, with axis the segment of the $x_{1}$-axis on which $x_{1} \leqq 8$, and with $K_{P} \supset \operatorname{Int}(\bar{K} \cup R \bar{K})$. One sees that

$$
K_{P} \supset T_{r}(K) \cup G^{\prime} \quad(r=1,2, \cdots) .
$$

The contraction a. This is a $C^{\infty}$-diff of $D$ onto $H^{\prime}$ which leaves $L^{\prime}$ pointwise invariant $\left[1\right.$, p. 274]. We infer that $\mathrm{a}(P) \in H^{\prime}-\mathrm{Cl} L^{\prime}$.

The reflection $t$. The point $Q$ is the intersection of the positive $x_{n}$ axis with $S$. Let $S_{Q}$ be an $(n-1)$-sphere with center $Q$ and diameter $\rho<1$, so small that $\phi \mid J S_{Q}$ reduces to the identity. Let $t$ be the reflection of $E-Q$ in $S_{Q}$ [1, p. 272].

The $C^{m}$-diff $\omega$. The domain of definition of $\omega$ includes $H^{\prime}-G^{\prime}$, and so is an open neighborhood of $a(P)$. Cf. p. 273 of [1].

The mapping $\omega_{e}$. By Lemma 5.1 of [1], the domain of $\omega_{e}$ includes $A$, and $\omega_{e}(x)=x$ on $A$.

The mapping $\sigma$. By Lemma 7.2 of [1] $\sigma$ is a $C_{P}^{m}$-diff of $C G^{\prime}$ into $E$. By this lemma and (2.6), $\sigma(x)=\omega_{e}(x)$ for $x \in C K_{P}$. Since $C K_{P} \subset A$ by (2.4), $\sigma(x)=x$ for $x \in C K_{P}$. Hence $P$ is a conical point of $\sigma$. 
The mapping $\lambda_{\omega} \mathrm{a}$. For present purposes $\lambda_{\omega} \mathrm{a}$ is a mapping for which (cf. (3.6) of [1])

$$
\left(\lambda_{\omega} a\right)(x)=\omega\left(a\left(\sigma^{-1}(x)\right)\right) \quad\left(x \in \sigma\left(D-G^{\prime}\right)\right) .
$$

We verify that $P$ is a conical point of $\lambda_{\omega} a$. The domain of $x$ in (2.7) is open. It contains $P$ since $P \in D-G^{\prime}$ and $\sigma(P)=P$. Now $\sigma^{-1}$ maps $\sigma\left(D-G^{\prime}\right)$, as a $C_{P}^{m}$-diff, onto $D-G^{\prime}$, with $P$ a conical point of $\sigma^{-1}$. Moreover a $\left(D-G^{\prime}\right)=H^{\prime}-G^{\prime}$, while $\omega$ operates as a $C^{m}$-diff on $H^{\prime}-G^{\prime}$. Returning to (2.7) observe that $\lambda_{\omega}$ a defines a $C_{P}^{m}$-diff of $\sigma\left(D-G^{\prime}\right)$ into $E$. It follows from Lemma 1.1 (ii) and (2.7) that $P$ is a conical point of $\lambda_{\omega} \mathbf{a}$.

Completion of Proof of Theorem 2.1. In accord with the line following (7.19) of [1] and line -13 on p. 275 in $[1], z=t(a(P))$. By $(7.22)^{\prime \prime}$ of $[1]$

$$
\Lambda_{\phi}(x)=t\left(\lambda_{\omega}(t(x))\right) \quad\left(x \in t\left(H^{\prime}\right)\right)
$$

(cf. [1, lines 2-3, p. 287]) so that if one sets $\mu(x)=\mathrm{a}^{-1}(t(x))$ for $x \in t\left(H^{\prime}\right)$

$$
\Lambda_{\phi}(x)=\left[t\left(\lambda_{\omega} \mathrm{a}\right) \mu\right](x) \quad\left(x \in t\left(H^{\prime}\right)\right) .
$$

We now apply Lemma 1.1. The $C^{\infty}$-diff $x \rightarrow \mu(x)$ of $t\left(H^{\prime}\right)$ into $E$ maps $z$ into $P$, since $z=t(a(P))$. From Lemma 1.1 and (2.9) we can then infer that $z$ is a conical point of $\Lambda_{\phi}$, since $P$ is a conical point of $\lambda_{\omega}$ a.

This establishes Theorem 2.1.

The generality of singularities of conical type is evidenced by the following theorem. Cf. [2].

TheOREM 2.2. Let $F$ be an arbitrary $C_{z}^{m}$-diffeomorphism into $E$ of an open subset $X \subset E$. There exists a $C_{z}^{m}$-diffeomorphism $F^{*}$ of $X$ into $E$ for which $z$ is a conical point and which is such that $F^{*}(x)=F(x)$ except at most in an arbitrarily small prescribed neighborhood of $z$.

\section{REFERENCES}

1. William Huebsch and Marston Morse, An explicit solution of the Schoenflies extension problem, J. Math. Soc. Japan vol. 12 (1960) pp. 271-289.

2. - Schoenflies extensions without interior differential singularity, Ann. of Math., to appear.

INSTITUTE FOR Advanced Study 